Presented at the 1977 Particle

Accelerator Conference, Chicago, IL,

March 16 - 18,1977

\title{
TRANSPORT OF INTENSE ION BEAMS
}

G. Lambertson, L. J. Laslett, and L. Smith

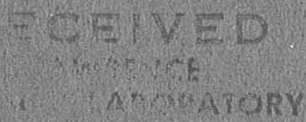

March 16, 1977

17) 221977

Prepared for the U. S. Energy Research and

Development Administration under Contract W-7405-ENG-48

\section{For Reference}

Not to be taken from this room

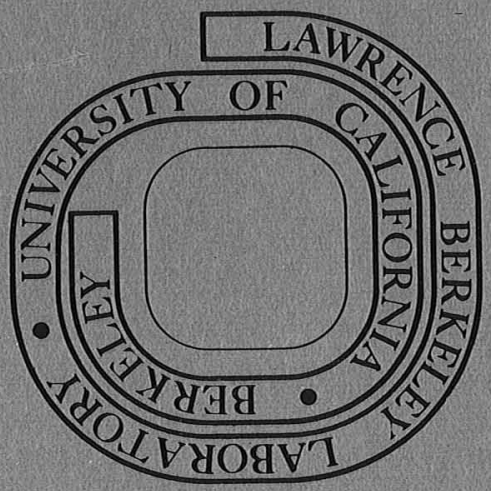




\section{LEGAL NOTICE}

This report was prepared as an account of work sponsored by the United States Government. Neither the United States nor the United States Energy Research and Development Administration, nor any of their employees, nor any of their contractors, subcontractors, or their employees, makes any warranty, express or implied, or assumes any legal liability or responsibility for the accuracy, completeness or usefulness of any information, apparatus, product or process disclosed, or represents that its use would not infringe privately owned rights. 


\section{Summary}

The possibility of using intense bursts of heavy ions to initiate an inertially confined fusion reaction has stimulated interest in the transport of intense unneutralized heavy ion beams by quadrupole or solenoid systems. We have examined this problem in some detail, using numerical integration of the coupled envelope equations for the quadrupole case. The general relations which emerge are used to develop examples of high energy transport systems and as a basis for discussing the limitations imposed by a transport system on achievable intensities for initial acceleration.

\section{Solution of the Envelope Equations}

The envelope equations of Kapchinskij and Vladimirskij (KV) are1).

$$
\begin{aligned}
& \frac{d^{2} a_{x}}{d s^{2}}=-k_{x}(s) a_{x}+\frac{\varepsilon^{2}}{\beta^{2} \gamma^{2} a_{x}{ }^{3}}+\frac{4 q^{2}}{A} \frac{N r_{p}}{\beta^{2} \gamma^{3}} \frac{1}{a_{x}+a_{y}} \\
& \frac{d^{2} a_{y}}{d s^{2}}=-K_{y}(s) a_{y}+\frac{\varepsilon^{2}}{\beta^{2} \gamma^{2} a_{y}^{3}}+\frac{4 q^{2}}{A} \frac{N r_{p}}{\beta^{2} \gamma^{3}} \frac{1}{a_{x}+a_{y}},
\end{aligned}
$$

where $a_{x, y}=$ beam half-width, height; $\pi \varepsilon=$ normalized emittance In either plane, $q$ and $A$ are the ion charge state and atomic weight, $\mathrm{N}$ is the number of particles per unit length, $r_{p}$ is the classical proton radius and

$$
\begin{aligned}
K_{x, y} & = \pm \frac{B^{\prime}(s)}{[B \rho]} \text { for quadrupoles } \\
& =\frac{1}{4}\left[\frac{B_{s}(s)}{[B \rho]}\right]^{2} \begin{array}{l}
\text { for solenoids (in a frame } \\
\text { rotating at the Larmor } \\
\text { frequency). }
\end{array}
\end{aligned}
$$

If $K(s)$ is a step-wise function of $s$, of constant magnitude or zero, Eqns. (1) can be put into dimensionless form by setting

$$
\begin{aligned}
& s=k^{-1 / 2} \theta \text { and } a=k^{-1 / 4} \varepsilon^{1 / 2}(\beta \gamma)^{-1 / 2} u: \\
& \frac{d^{2} u_{x}}{d \theta^{2}}=-S_{x}(\theta) u_{x}+\frac{1}{u_{x}{ }^{3}}+\frac{Q}{u_{x}+u_{y}} \\
& \frac{d^{2} u_{y}}{d \theta^{2}}=-s_{y}(\theta) u_{y}+\frac{1}{u_{y}{ }^{3}}+\frac{Q}{u_{x}+u_{y}},
\end{aligned}
$$

where $Q=\frac{4 q^{2}}{A} \frac{N r_{p}}{B \gamma^{2} \varepsilon K^{1 / 2}}$ and $S(\theta)$ is a step function of unit amplitude. If $S(\theta)$ is periodic, the necessary aperture and the current for a matched beam can be expressed as functions of Q. For quadrupoles,

\footnotetext{
* Work supported by the U.S. Energy Research and Development Administration.
}

$$
\begin{aligned}
& a=C_{1}\left(\frac{A}{q}\right)^{1 / 3} B_{Q}^{-1 / 3}(B \gamma)^{-1 / 3} \varepsilon^{2 / 3} u_{m}{ }^{4 / 3}, \\
& I=C_{2}\left(\frac{A}{q}\right)^{1 / 3} B_{Q}^{2 / 3}(B \gamma)^{5 / 3}(\varepsilon)^{2 / 3} u_{m}^{4 / 3} \frac{Q}{u_{m}^{2}} \\
& =\frac{C_{2}}{C_{1}}(\beta \gamma)^{2} B_{Q} a \frac{Q}{u_{m}^{2}} \text {, } \\
& \text { where } c_{1}=\left(\frac{m_{p} c}{e}\right)^{1 / 3}=1.46 \quad \text { [MKSA units] } \\
& C_{2}=\frac{1}{4}\left(\frac{4 \pi}{\mu_{0}}\right)^{5 / 6}\left(\frac{m_{p} c^{2}}{r_{p}}\right)^{1 / 6}=3.66 \times 10^{6} \\
& a=C_{3}\left(\frac{A}{q}\right)^{1 / 2} B_{s}^{-1 / 2} \varepsilon{ }^{1 / 2} u \\
& I=C_{4} B_{s}(\beta \gamma) \varepsilon\left(u^{2}-\frac{1}{u^{2}}\right)=\frac{C_{4}}{C_{3}^{2}}\left(\frac{q}{A}\right)(\beta \gamma) B_{s}{ }^{2} a^{2}\left(1-\frac{1}{u^{4}}\right) \\
& \text { where } c_{3}=\left(\frac{2 m_{p} c}{e}\right)^{1 / 2}=2.5 \\
& C_{4}=\frac{1}{4}\left(\frac{4 \pi}{\mu_{0}}\right)=2.5 \times 10^{6} \text {. }
\end{aligned}
$$

Q, or the corresponding $u_{m}$ or $u$, can be regarded as a free parameter measuring the influence of the spacecharge force on particle motion. In the quadrupole case the relation between $Q$ and $u_{m}$ depends on the lattice structure. It is convenient to use the phase

advance per period, $\mu=\int \frac{d s}{\beta}=\int \frac{d \theta}{u_{x}{ }^{2}}=\int \frac{d \theta}{u_{y}{ }^{2}}$, as the

space charge parameter, since it has a more immediate physical significance than $Q$ or $u_{m}$. In Fig. 1 is plotted $Q / u_{m}^{2}$ and $u_{m} 4 / 3$ for a FODO lattice with equal drift and magnet lengths and a phase advance per period of $120^{\circ}$ at zero intensity. It is evident from the figure and from the form of Eqns. (4) for the solenoid case that, on the basis of these simple considerations, there is no limit to the current which can be transported, provided that the aperture can be made large enough and the variation in individual particle motion with intensity is tolerable. 


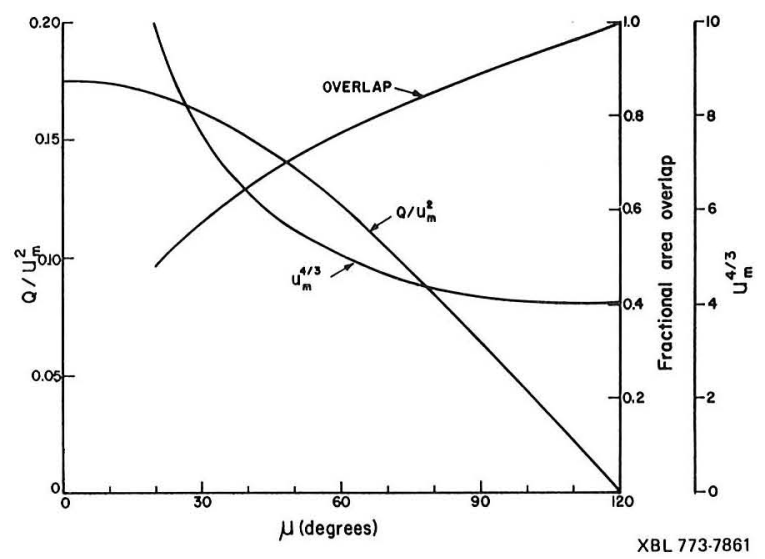

Transport at High Energy

As an application of these equations, we consider a situation in which a beam is extracted from an accelerator, passed through a buncher and allowed to drift some distance to shorten the pulse and increase the current to meet the targeting requirements. It is assumed that the rate of increase of current with distance is sufficiently slow that the transverse motion will adjust itself adiabatically to the matched conditions if it is matched at the entrance to the channel, where the current is low. We further assume that the elements at the end which focus the beam onto the pellet are adjusted to accept the phase-space configuration of the peak of the current pulse, which requires that there be a substantial overlap of the phase space ellipses for peak and lower intensities. The quantity, $n$, also shown in Figure 1, is the fraction of the zero intensity phasespace area lying inside the higher intensity ellipse, assuming an emittance independent of intensity. It can be seen that requiring $n$ to be larger than, say, $50 \%$ sets a definite limit on peak current for a given quadrupole field. Table I gives four examples: energy, peak current and emittance are target requirements ${ }^{2}$, $\mathrm{B}_{0}$ was chosen arbitrarily for $U_{238}^{+}$and as high as seemed realistic for $I^{\dagger} 27$ because of the constraint on $n$.

\section{TABLE I}

\section{Examples of High Energy Transport}

\begin{tabular}{lccccc} 
Ion Type & \multicolumn{2}{c}{$U_{238}^{+1}$} & & \multicolumn{2}{c}{$I_{127}^{+1}$} \\
\cline { 2 - 3 } \cline { 5 - 6 } Energy (GeV) & 100 & 40 & & 40 & 10 \\
$I_{\text {peak }}(\mathrm{KA})$ & 3.0 & 1.25 & 7.5 & 5 \\
$\varepsilon\left(10^{-5}\right.$ m-radians $)$ & 4.0 & 1.8 & 3.0 & 1.6 \\
$\mathrm{~B}_{\mathrm{Q}}(\mathrm{T})$ & 1 & 1 & 3 & 3 \\
$\mathrm{a}(\mathrm{cm})$ & 4.2 & 3.0 & 2.1 & 2.6 \\
$\mu(\mathrm{deg})$ & 107 & 100 & 92 & 44 \\
$\eta$ & .95 & .92 & .89 & .67
\end{tabular}

Solenoid focusing does not look favorable for the cases considered. It is not difficult to show that $\eta=1-\frac{4}{\pi} \tan ^{-1} \frac{u-1}{u+1}$, whence $u<2.5$ for $n>.50$.
Eqns. (4) then demand very high fields and large apertures.

\section{Transport at Low Energy}

Equations (3) and (4) indicate that particle current must be much reduced at lower energy. Hence, to provide a final high current, the accelerator system is required to build up the current by orders of magnitude by some combination of stacking in transverse space and longitudinal compression.

Additional considerations will affect the application of Eqns. (3) and (4). We assume that there is no need to transport a reduced current as well as the highest current through the same system, or equivalently, that the lower current portion may have a lower emittance. This would then permit the zero-intensity phase shift $\mu_{0}$ to approach the pass-band 1 imit of $180^{\circ}$ and $\mu$ to be made as small as allowed by the aperture or other considerations. However, a strong field $B_{0}$ may result in quadrupole lengths and drift lengths that are too short, relative to the aperture, to permit fields that are reasonably linear and defined in length (as was assumed in the analysis). For the strong quadrupole case, then, we introduce the additional requirement that the ratio of aperture radius to quadrupole length not exceed a limiting value $R$ and this results in the following limit on particle current in the FODO lattice with equal drift and quadrupole lengths:

$$
I=C_{s}\left(\frac{A}{q}\right)(B \gamma)^{3} R^{2} \frac{\Theta^{2} Q}{u_{m}^{2}},
$$

where $c_{5}=\frac{1}{16}\left(\frac{4 \pi}{\mu_{0}}\right)^{1 / 2}\left(\frac{m_{p} c^{2}}{r_{p}}\right)^{1 / 2}=1.9 \times 10^{6}$

and $2 \Theta$ is the cell length in the scaled variable, $\theta$.

The quantity $\Theta^{2} \frac{Q}{u_{m}{ }^{2}}$ will depend on the phase ad-

vances, but has a maximum value close to unity.

Two other limitations should be kept in mind. First, the electrostatic potential in the beam can become comparable to the kinetic energy and, second, if $B_{S}$ or $B_{0}$ approaches $\frac{2[\mathrm{Bp}]}{\mathrm{a}}$, ions entering a lens at radius $a$, will be turned back at low intensity for a quadrupole and at any intensity for a solenoid. Both potential/ kinetic energy and $\mathrm{Ba} / 2[\mathrm{Bp}]$ must be much less than unity for the paraxial ray approximation used in this paper to be valid.

For a numerical example, we consider a beam of $\mathrm{U}^{+} 3 \mathrm{~s}$ at a kinetic energy of $1.0 \mathrm{MeV}$ and the same normalized emittance as the 100-GeV example of Table I. With a strong $B_{0}$ and no restriction on $R$, the first column of Table II shows that a current of 4.65 amperes can be transported by the F0D0 system. Restricting $R$ to about 0.5 . reduces the current to 1.0 ampere (column $2)$; with reduced $\mathrm{B}_{0}$, a better compromise is found at 2.42 ampere in column 3 but with somewhat larger aperture roughly proportional to the ratio $I / B_{0}$ in consistency with Eqns. (3) and the approximate constancy of $\mathrm{Q} / \mathrm{u}_{\mathrm{m}}^{2}$. 
TABLE II

\begin{tabular}{lccr} 
Ion Type & \multicolumn{3}{c}{$\mathrm{U}_{238}^{+1}$} \\
Energy (MeV) & \multicolumn{1}{c}{1.0} \\
$\varepsilon\left(10^{-5}\right.$ m-radian) & \multicolumn{3}{c}{4.0} \\
\cline { 2 - 4 } $\mathrm{B}_{Q}$ (tes1a) & 3.0 & 3.0 & 1.5 \\
$\mathrm{R}$ & 0.68 & 0.52 & 0.50 \\
$\mathrm{I}$ (Ampere) & 4.65 & 1.0 & 2.42 \\
$\mathrm{a}$ (cm) & 41.4 & 21.5 & 45.0 \\
$\mu_{0}$ (deg) & 160.0 & 120.0 & 160.0 \\
$\mu$ (deg) & 35.3 & 87.7 & 44.7
\end{tabular}

At this energy, the solenoid becomes comparable in effectiveness with quadrupoles. A choice between the two will depend on special features of the transport problem to be solved.

\section{Critique of the Envelope Equation Approach}

Although the phase-space distribution underlying the KV envelope equations is not very realistic, the results are known to provide a useful guide for moderate intensities. However, since we are interested in understanding beam behavior under extreme space charge conditions, we have investigated a number of effects not described by Eqns. (1).

a) Incoherent image forces should be taken into account in any case other than that of a round beam in a round pipe, since both economic and focusing field strength limitations demand that these beams substantially fill the vacuum channel. A simple, if somewhat academic, test can be made by assuming an elliptical chamber, confocal with an elliptical beam; in this situation the image forces are linear,3) but change the functional form of the space charge terms in Eqns. (1). It was found that even if the chamber coincides everywhere with the beam edge, the relations described by Fig. (1) change very little. In the more realistic case of an elliptical beam in a round pipe, we have found by numerical computation that the non-1 inear part of the image fields is small compared to nonlinear fields due to a degree of non-uniformity one might reasonably expect in the charge distribution.

Coherent image forces, which attract the beam to the enclosing pipe, come into play if the beam is steered improperly or is deflected by a lens placement error. We find that a coherent motion of the beam is stable, but with a reduction in phase advance comparable to the reduction in the incoherent motion.

b) For a round beam, born in a field free region but transported by a continuous solenoid, there exists an infinity of stationary self-consistent phase space distributions in addition to the KV distribution. We have examined a broader class of these distributions than in previous work 4 and find that in all cases the spatial distribution tends toward uniformity with increasing current, although the individual particle motion becomes highly non-linear except for the KV distribution. Moreover, the dependence of current and aperture on $A / q, B_{S}$, $B \gamma$ and $\varepsilon$ is as given in Eqns. (4), independent of the form of the distribution function in the high-intensity limit. It is not clear to us, however, whether any of the distributions examined offers a better description of a real beam than the
$\mathrm{KV}$ distribution. It is known that a large sub-class of these distributions is stable for small perturbation of initial conditions, 5 ) but we found that the KV distribution is unstable, with a threshold in current, for a large number of modes (see also Ref. 4). The lowest threshold occurs at $u=1.6$, which would imply that Eqns. (4) have a very limited range of validity. On the other hand, the KV distribution has special mathematical properties and we prefer to believe that Eqns. (4) are probably qualitatively correct for more realistic distributions which are probably stable.

c) Since the Hamiltonian is not a constant of the motion for a quadrupole transport line, it is not possible to construct stationary (i.e., periodic) solutions by the technique outlined in the previous paragraph. As a partial step away from the KV distribution we examined a "self-inconsistent" problem by tracing individual particle trajectories in a field with a linear part generated by the periodic solution of the envelope equation, plus cubic terms appropriate to a parabolic density profile of the same outer dimensions. For intensities such that $\mu$ is less than $\sim 20^{\circ}$, we find a large growth in amplitude of some particles and the development of an island structure in their phase space, indicative of resonant behavior in the periodic non-linear field. We are thereby led to suspect that a quadrupole transport system may be subject to unstable behavior at high intensity.

\section{References}

1) I.M. Kapchinskij and V.V. Vladimirskij, Proc. Internat. Conf. on High Energy Accelerators, CERN 1959, p. 274.

2) Final Report of ERDA Summer Study of Heavy Ions For Inertial Fusion, LBL-5543, p. 14-15 (Dec. 1976).

3) E. Regenstreif, CERN/PS/DL 76-4, June 1976.

4) Proc. 1970 Proton Linac Conference (NAL); R.L. Gluckstern, p. 811 and R.L. Gluckstern, R. Chasman, K. Cranda11, p. 823.

5) R.C. Davidson and N.A. Kra11, Phys. Fluids 13, 1543 (1970). 
This report was done with support from the United States Energy Research and Development Administration. Any conclusions or opinions expressed in this report represent solely those of the author(s) and not necessarily those of The Regents of the University of California, the Lawrence Berkeley Laboratory or the United States Energy Research and Development Administration. 